\title{
Nitriding of AISI 304 Stainless Steel by PIII in DC and RF Toroidal Discharges
}

\author{
R. Valencia ${ }^{a}$, R. López-Callejas ${ }^{a, b}$, A. Muñoz-Castro ${ }^{a}$, S.R. Barocio ${ }^{a}$, \\ E. Chávez $\mathrm{A}^{a}$, and O. Godoy-Cabrera ${ }^{a, b}$ \\ ${ }^{a}$ Instituto Nacional de Investigaciones Nucleares, Plasma Physics Laboratory, \\ Apartado Postal No.18-1027 Col. Escandón, C. P. 11801, México D.F., Mexico \\ ${ }^{\mathrm{b}}$ Instituto Tecnológico de Toluca, Electronics Department, \\ Apartado Postal 890, Toluca, Mexico
}

Received on 5 December, 2003; revised version received on 30 April, 2004

\begin{abstract}
Plasma immersion ion implantation (PIII) of stainless steels with nitrogen has been successfully used for surface hardening purposes. This process has been carried out inside a toroidal discharge chamber in a DC/RF plasma. The RF plasma was created by one antenna located inside the chamber, diametrically opposite to the DC electrode. The latter is polarized with $1 \mathrm{kV}$ and then the discharge is controlled by varying the gas pressure before the RF signal is applied. The main plasma parameters were established by means of double electric probes yielding electron temperature values within $0.5-1.5 \mathrm{eV}$ and density values within $1.5 \times 10^{15}$ to $4 \times 10^{15}$ $\mathrm{m}^{-3}$ for the DC case while $1.5-3.0 \mathrm{eV}$ and $7 \times 10^{14}$ to $3 \times 10^{15} \mathrm{~m}^{-3}$ were reached with RF assisted DC. We present in this work the experimental results obtained from a PIII process applied to AISI 304 stainless steel plates. The outcome shows that the Vickers hardness has been incremented according to the gas pressure within the $1 \times 10^{-1}$ to $1 \times 10^{-3}$ mbar range. The treated plates were analyzed by scanning electron microscopy (SEM) and the results point to an increased percentage of nitrogen, around $20 \%$. By means of x-ray diffractometry (XRD) the gamma expanded phase and compounds such as $\mathrm{Fe}_{3} \mathrm{NiN}, \mathrm{Ni}_{4} \mathrm{~N}$, FeNiN and $\mathrm{Fe}_{3} \mathrm{~N}$ were determined.
\end{abstract}

\section{Introduction}

Plasma immersion ion implantation (PIII) is an effective surface modification method for treating complex shape components [1-7]. In PIII, the nitrogen ions are extracted directly from the plasma in which the samples to be processed are immersed. This is achieved by applying negative high voltage pulses of $1-100 \mathrm{kV}$, of 1-100 $\mu$ sec duration and 100$1000 \mathrm{~Hz}$ repetition frequency to the sheath formed between the plasma and the sample structure. In order to achieve a thicker nitrided layer in metals, a treatment at higher temperature is usually preferred. The targets are directly heated by the high incident ion-flux during PIII, and the target temperature can be controlled by regulating the frequency and duration of the high-voltage pulses applied to the samples. This process combines conventional ion implantation and diffusion.

Austenitic stainless steels, such as AISI-304, present a relatively low hardness, in the order of 200-300 HV. There are several processes intended to increase the surface hardness and tribological quality without reducing the excellent corrosion resistance that makes these steels so attractive in many applications. Plasma nitriding at temperatures above $500^{\circ} \mathrm{C}$, while giving significant improvement in wear resistance, tends to affect adversely the corrosion performance of stainless steels due to the precipitation of $\mathrm{CrN}$ which removes $\mathrm{Cr}$ from its solid solution [8].

According to the so called Tian theory [9], in a typical
PIII process, ions within the sheath are accelerated to thousands of $\mathrm{eV}$ resulting in a transient time in the order of nanoseconds; so that the sheath transient time is practically instantaneous when compared to the voltage pulse, typically in the range of msec. Under low pressure conditions $\sim 10^{-3}$ mbar range, collisionless conditions are valid and all ions within the sheath do not undergo collisions or charge transference and are implanted at the applied voltage. Since the sheath voltage is always greater than the electron temperature, we can assume that a quasi-static Child-Langmuir law sheath exists at all times and that the ion current is spatially constant within the sheath. As a result, when a negative voltage is applied to the target, a dynamic process will commence. The primary electrons are pushed away, an ion sheath forms and ions are implanted into the target at an applied voltage that varies at the beginning, depending on the slew rate of the power modulator.

The influence of the vacuum chamber geometry on many of the above mentioned processes can be considerable. Thus, for instance, in contrast to the most commonly used cylindrical geometry, in a toroidal one there are no end losses of plasma particles [10] owing to the application of equally toroidal magnetic fields in configurations close to those typical of nuclear fusion devices [11]. Due to this and similar potential uses of toroidal chambers, we are developing a line of research within our PIII programme with a view to reducing diffusion losses by means of toroidal magnetic confinement. 


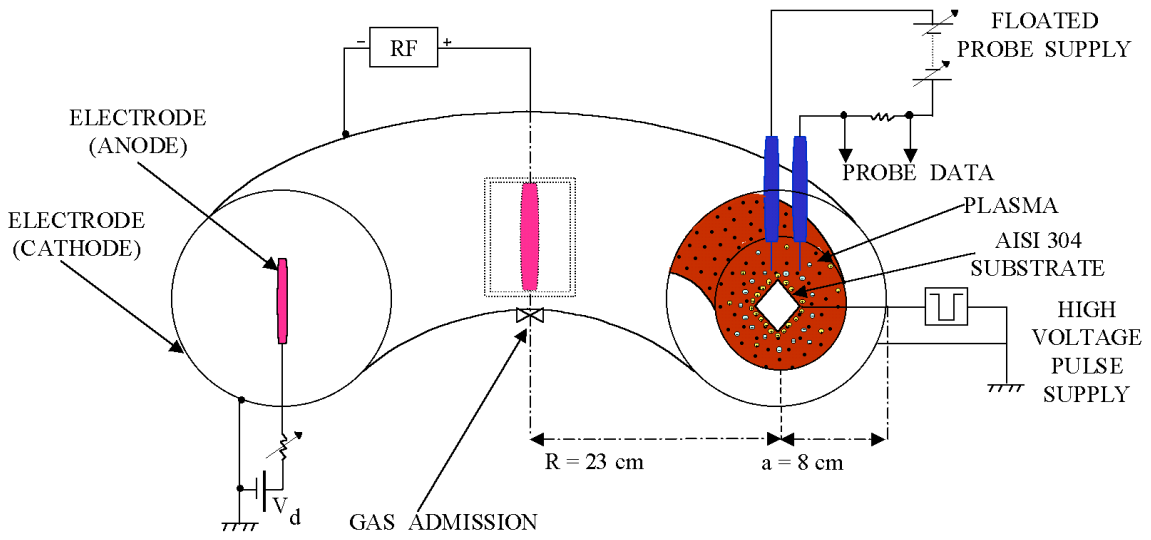

Figure 1. Cross-sectional view of the toroidal chamber, displaying the DC and RF supplies, plasma, probe and high voltage pulse supply.

\section{Experimental Set-Up}

The toroidal vacuum chamber selected for this work is 23 $\mathrm{cm}$ in major radius and $8 \mathrm{~cm}$ in minor radius, as shown in Fig. 1. Glow discharges in nitrogen are produced between a cylindrical stainless steel electrode (anode) and the grounded toroidal chamber wall. The anode is inserted within the toroidal chamber through a vertical port and it is connected to a $0-1500 \mathrm{~V} / 2 \mathrm{~A}$ DC power supply through a $488 \Omega$ load resistor intended to limit the current flow to the plasma. The negative terminal of the power supply and the toroidal chamber were grounded. The highest discharge current used in the experiment was $500 \mathrm{~mA}$. By assuming that all the wall is exposed to the discharge, we can roughly estimate a maximum current density of $70 \mu \mathrm{A} / \mathrm{cm}^{2}$.

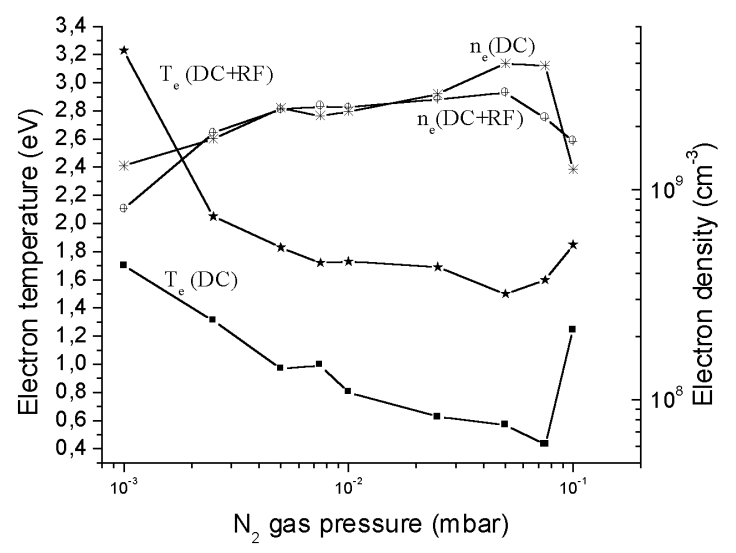

Figure 2. Dependence of the plasma density and temperature on nitrogen gas pressure.

The plasma sources we have chosen are a highly stable DC glow discharge and a $13.56 \mathrm{MHz}$ RF generator positioned at 180 degrees from the anode DC source. A schematic view of the PIII setup is shown in Fig. 1. The plasma in the toroidal chamber was extensively characterized both in density and temperature applying the Auciello double probe method for different pressures as described in [12]. Fig. 2 shows the plasma density and the electron temperature as functions of the nitrogen pressure, measured near the torus axis where the density turned out to be higher, in two plasma conditions, one under a 350V/0.2A DC only and the other under a $350 \mathrm{~V} / 0.2 \mathrm{~A} \mathrm{DC}+50 \mathrm{~W} \mathrm{RF}$, the density remaining practically the same in both regimes. In such an axial neighborhood, $n_{e}$ is greater due to locally longer electric field lines which favor ionization by collisions. For the same reason, $n_{e}$ is increased with the working pressure, whereas $T_{e}$ is decreased as observed in Fig. 2. Our results seem to be in good agreement with the typical behavior in density and temperature reported by Kojima and Park [13-14]. The sudden drop in the electron density at pressures higher than $1 \times 10^{-1}$ mbar can be attributed to a lesser degree of ionization in the gas.

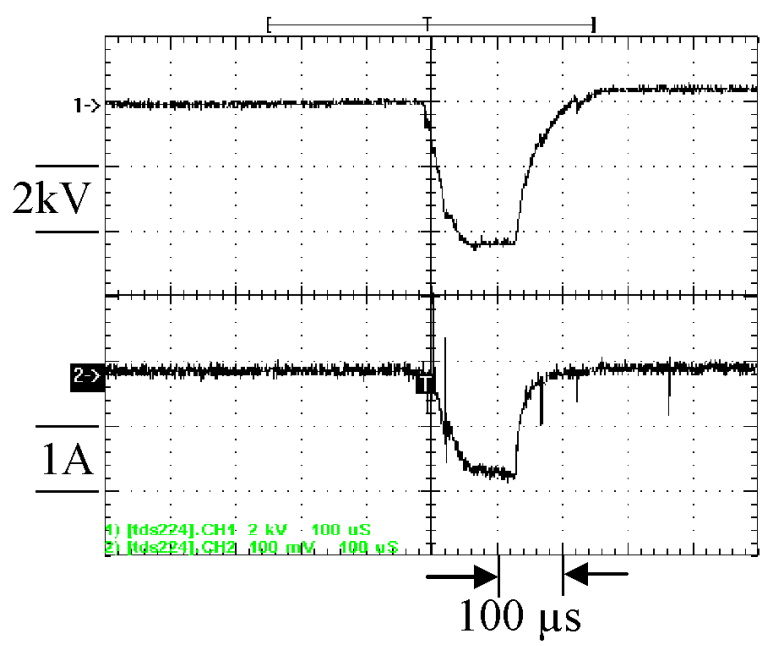

Figure 3. Implantation voltage and current waveforms: (1) voltage and (2) current.

The implantation voltage and target ion current waveforms are displayed in Fig. 3. The rise or fall time depends on the inherent capacitance of the hardware and plasma sheath, and, as a result, there is a current surge when the pulse modulator is switched on and off. In order to measure the waveforms, a high voltage probe was used along with a wide band current transformer with a 0.1 output volts/amp terminated with $50 \Omega$, all the set being electrostatically shielded. 
In Fig. 3, the horizontal scale corresponds to $100 \mu$ s per division. The vertical scales are $2 \mathrm{kV}$ and $1 \mathrm{~A}$ per division, respectively. We present some results obtained from the PIII process applying $-5 \mathrm{kV}$ bias pulses with a half width 100 $\mu$ s long and a $1 \mathrm{kHz}$ frequency during 4 hours at different pressures within the toroidal vacuum chamber.

\section{Results}

The process was carried out on AISI 304 stainless steel samples within a pressure range between $10^{-1}$ to $10^{-3}$ mbar and a 1.8 A current demanded from the sample. The corresponding waveforms are displayed in Fig. 3. The sample temperature remained between $350^{\circ} \mathrm{C}$ and $450^{\circ} \mathrm{C}$ during the whole PIII process, at all the work pressures. Once the process is finished, the surfaces of the samples show a distinct roughness, not only because of the grain boundary etching but also of the deformation of the grains due to their higher nitrogen content [2]. In order to establish the degree of surface modification of the samples, these were characterized by means of scanning electron microscopy (SEM) as well as Vickers microhardness testing.

The phase transformation in the near surface is responsible for the change in the sample appearance, as indicated by XRD results in Fig. 4. We have used a diffractometer with $\mathrm{CuK} \alpha$ radiation operated at a $35 \mathrm{kV}$ acceleration and a $25 \mathrm{~mA}$ current in order to determine the phases present in the modified layer. The analysis of the standard reference sample is shown in Fig. 4a, covering a $2 \theta$ interval between $30^{\circ}$ and $100^{\circ}$. The most relevant information is found at $43.4^{\circ}$, $50.6^{0}, 74.6^{0}, 90.5^{0}$ and $95.7^{0}$ C. Figs. 4.(b)-4.(d) exhibit the diffractogrammes taken from one PIII treated sample at different gas pressure intervals. In these figures, some additional peaks are observed at: i) the angles $41.2^{\circ}$ and $48.1^{0}$ where roaldite $\left(\mathrm{Fe}_{3} \mathrm{NiN}\right)$ exists; ii) the angles $41.8^{\circ}, 48.5^{\circ}$ and $71.4^{0}$ where nickel nitride $\left(\mathrm{Ni}_{4} \mathrm{~N}\right)$ is found; iii) the angles $40^{\circ}, 45.4^{0}, 49^{0}, 85.5^{\circ}$ and $86.1^{0}$ where iron nickel nitride $(\mathrm{FeNiN})$ is present, and iv) the angles $38.4^{0}, 69.6^{0}$, $77.1^{0}, 84.2^{0}, 86.1^{0}$ and $96.5^{\circ}$ where iron nitride $\left(\mathrm{Fe}_{3} \mathrm{~N}\right)$ is located. In the same Fig. 4.(b)-4.(d), the peaks associated to the original AISI 304 stainless steel can be observed.

The broad peaks labelled $\gamma_{N}$ at a $2 \theta$ angles smaller than those of the untreated stainless steel sample are associated with the nitrogen rich expanded austenitic phase. $\gamma_{N}$ is a metastable phase consisting of supersaturated nitrogen in a solid solution. This is a typical result in which it is possible to see the peaks corresponding to the $\gamma_{N}$ phase at $2 \theta$ values $41^{\circ}$ and $48^{\circ}$ as the more intense and with (111) and (200) preferential orientations, respectively. The relative intensity of the $\gamma_{N}$ phase is similar to and sometimes greater than that of primary austenite indicating that $\gamma_{N}$ is thick and abundant in the near surface layer.

The thickness of the modified layer is estimated by cross-sectional metallography. The scanning electron micrograph in Fig. 5 shows that the thickness is approximately $4 \mu \mathrm{m}$ and the modified layer is very homogeneous and uniform in thickness. The polyhedric structures on the right hand side of the non nitrided layer can be identified with the grain of the untreated steel while the dark dots are mainly formed by carbon impurities.

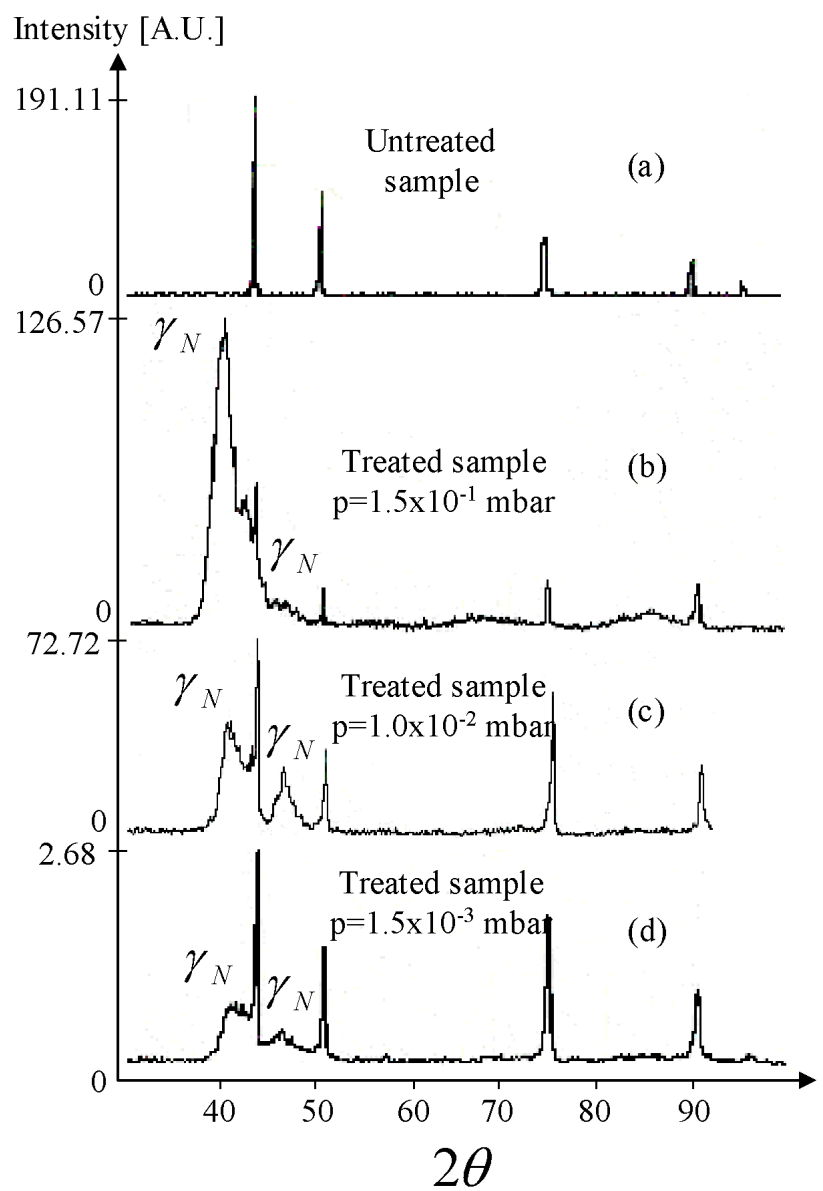

Figure 4. Difractogrammes of treated and untreated samples at different pressures. Amplitudes are scaled in arbitrary units.

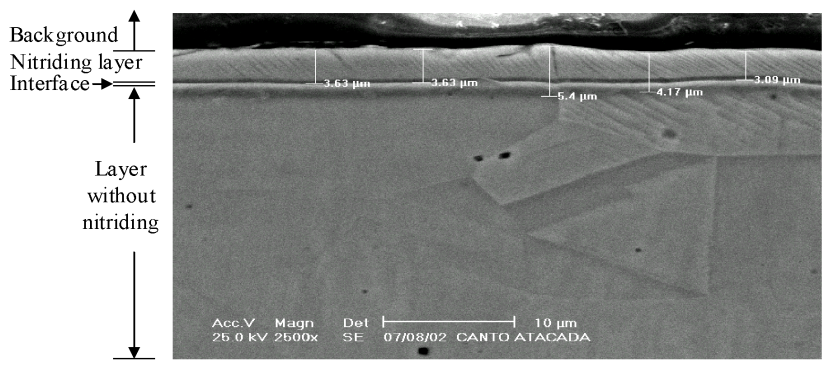

Figure 5. Cross-sectional scanning electron microscopy (SEM) micrograph of the treated AISI-304 stainless steel.

The main mechanical tests on the AISI 304 steel samples were Vickers microhardness measurements. To this purpose, a tester with 15, 25, 50, $100 \mathrm{~g}$ loads was applied during 10 second periods. The results, expressed in Vickers units (HV), represent the resistance offered by steel to plastic deformation by penetration. The microhardness measurement that results from the PIII treatment at various pressures is displayed in Fig. 6. The maximum microhardness values, near 750 for $15(\mathrm{~g})$, is higher than that reported by Tian [15]. The surface microhardness diminishes with an increasing indentor load. However, a smooth decline of the curve means that the hardness value is not very sensitive to 
the load and suggests the presence of a thick modified layer, as showed in Fig. 5. From the results displayed in Fig. 6 follows that the best microhardness conditions are achieved at a $1.5 \times 10^{-1}$ mbar gas pressure in the chamber. Clearly, then, the nitrogen implanted steel hardness is increased with respect to the standard untreated steel sample as well as samples treated in cylindrical chambers [16].

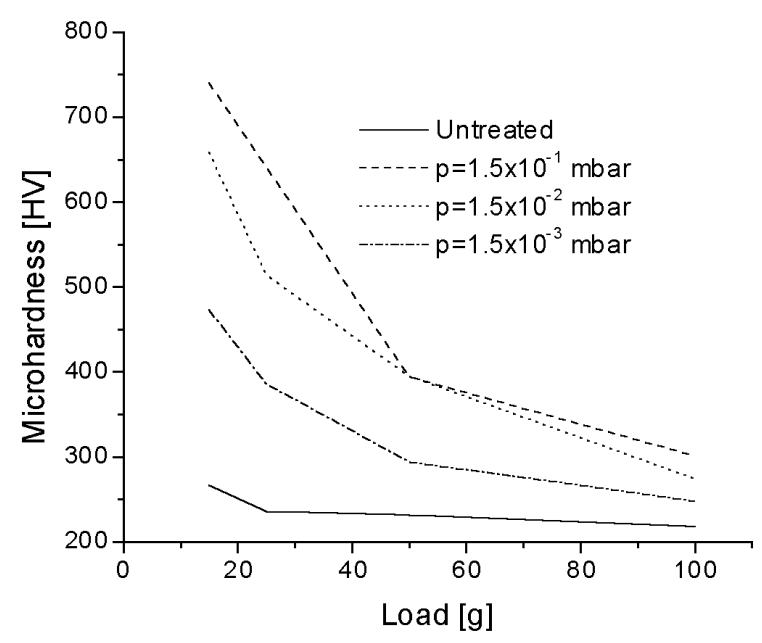

Figure 6. Relationship between surface microhardness and indentation load for the PIII treated AISI-304 stainless steel with respect to the untreated reference steel sample.

\section{Conclusions}

A PIII experimentation on a DC/RF plasma has been carried out within a toroidal discharge chamber given the potential advantages of this geometry, in terms of longer electric field lines, over its more conventional cylindrical alternatives. The hardness of PIII treated AISI-304 stainless steel has been increased several times, compared to the untreated samples, due to the lattice expansion created by the supersaturation of nitrogen. This hardness increase is the most important contribution of our experiment.

We have obtained excellent results for low voltage PIII as to the improvement of microhardness. XRD data indicate that the AISI-304 sample surface has a uniform layer composed of expanded austenite. These results can be attributed to, among other factors, the adequate temperature control on the samples throughout the implantation process. Thus, keeping a steady range below $500^{\circ} \mathrm{C}$ allowed us to improve substantially the tribological properties of the steel without spoiling its considerable corrosion resistance, associated to its $\mathrm{Cr}$ content.

High flux ion implantation raises the modification efficiency overcoming surface barriers and building up a high nitrogen concentration, rapidly enough to facilitate dopant diffusion. It is noteworthy that, because the critical load can be considerably improved by this process, there exists a great industrial potential in it.

\section{Acknowledgements}

This work was partially supported by CONACYT under contract 39676-Y and by COSNET under contract 642.0203-PR. The authors wish to thank the technical collaboration by M. T. Torres M. and I. Contreras V.

\section{References}

[1] J. R. Conrad, J. L. Radtke, R. A. Dodd, and F. J. Worzala, J. Appl. Phys. 62, 4591 (1987).

[2] G. A. Collins, R. Hutchings, K. T. Short, J. Tendys, X. Li, M. Samandi. Coat. Technol. 74-75, 417 (1995).

[3] C. Blawert and B.L. Mordike, Surf. Coat. Technol. 116-119, 352 (1999).

[4] X. B. Tian, Z. M. Zeng, B. Y. Tang, and P. K. Chu, Thin Solid Films 366, 150 (2000).

[5] X. B. Tian, Y. X. Leng, T. K. Kwork, L. P. Wang, B. Y. Tang, and P. K. Chu, Surf. Coat. Technol. 135, 178 (2001).

[6] M. Ueda, G. F. Gomes, L. A. Berni, J. O. Rossi, J. J. Barroso, A. F. Beloto, E. Abramof, and H. Reuther, Nucl. Instr. and Meth. Phys. Res. B 161-163, 1064 (2000).

[7] I. H. Tan, M. Ueda, R. S. Dallaqua, J. O. Rossi, A. F. Beloto, E. Abramof, Y. Inoue, and O. Takai, Plasma Sources Sci. Technol. 11, 317 (2002).

[8] Z. L. Zhang and T. Bell, Surf. Eng. 1, 131 (1985).

[9] X. Tian, B. Tang, and P. K. Chu, J. Appl. Phys. 86, 3567 (1999).

[10] B. C. Zhang and R. C. Cross J. Vac. Sci. Technol. A 16 (4), 2016 (1998).

[11] K. Hiramatsu and S. Takamura, Jpn. J. Appl. Phys. 31, 2243 (1992).

[12] O. Auciello and D:L: Hamn, Plasma Diagnostic, Vol I, Discharge Parameters and Chemistry, Academic Press, New York, (1989).

[13] H. Kojima, H. Kako, M. Terada, H. Sugar, and T. Okuda, Jpn. J. Appl. Phys. 24, 1432 (1985).

[14] Ch. H. Park, Y. M. Sung, and W. G. Lee, Thin Solid Films 312, 182 (1998).

[15] X. B. Tian, Z. M. Zeng, B. Y. Tang, T. K. Kwork, and P. K. Chu, Surf. Coat. Technol. 128-129, 226 0.2in(2000).

[16] R. López-Callejas R. Valencia-Alvarado, A. E. MuñozCastro, O. G. Godoy-Cabrera, S. R. Barocio, and G. Hernandez V, ASM International Proc. 2nd Surf. Eng. Cong \& Exp., to be published (2003). 